\title{
Compact Double-Sided Modular Linear Motor for Narrow Industrial Applications
}

\author{
Loránd Szabo, Dan-Cristian Popa, Vasile Iancu \\ Department of Electrical Machines, Technical University of Cluj, Romania
}

\begin{abstract}
Linear drive technologies are steadily expanded in various applications, especially in industry, where high precision electrical direct drive systems are required. In this paper a double-sided variant of a novel direct driven modular permanent magnet linear motor is presented. Its characteristics are computed by means of 3D FEM magnetic field analysis. An interesting industrial application in which it can be used is also presented.
\end{abstract}

\section{INTRODUCTION}

The field of linear electrical machines is widely expanding. Numerous practical applications in diverse areas having dynamic markets have already taken in study. This tendency is expected to continuously increase in the coming years [1]. Therefore any new results in this field could be of real interest for the specialists round the world.

The transverse flux motors (TFM) are relatively newcomers in the class of the special electric machines. They offer very high force/volume densities, but generally have complicated construction with sophisticated 3D flux paths. Therefore their iron core must be constructed of expensive soft magnetic composites (SMC).

In this paper a new modular permanent magnet linear motor is presented and analyzed by means of $3 \mathrm{D}$ finite elements method (FEM) based numeric field computation.

The proposed linear motor structure in spite of its transverse magnetic flux machine characteristics is relatively simple and can be built up of low cost materials (the mover of classical steel sheets and the platen of massive iron), avoiding the use of expensive SMC [2].

Its double-sided structure eliminates the high attracting forces between the stator and the moving armature of the linear machine. As its coil's end-windings are placed in the direction of the movement they are very compact and thin, hence they can be used also in very narrow places.

In the paper also the double salient permanent magnet linear motor from which this linear TFM structure is derived is presented.

The direct linear drives similar to that presented in the paper become widely used in high performance industrial equipment because they are combining high force development with precise positioning capabilities. High precision manufacturing processes (mainly the semiconductor fabrication) were the first ones taking the advantage of direct driven linear positioning systems [3].

In the final part of the paper a high precision industrial linear positioning system directly driven by double-sided modular linear motors is presented.

\section{Permanent Magnet Linear Motor Variants}

Several years ago the already classical hybrid linear stepper motor (see Fig. 1) was considered to be one of the best solutions for high precision linear positioning systems [4].

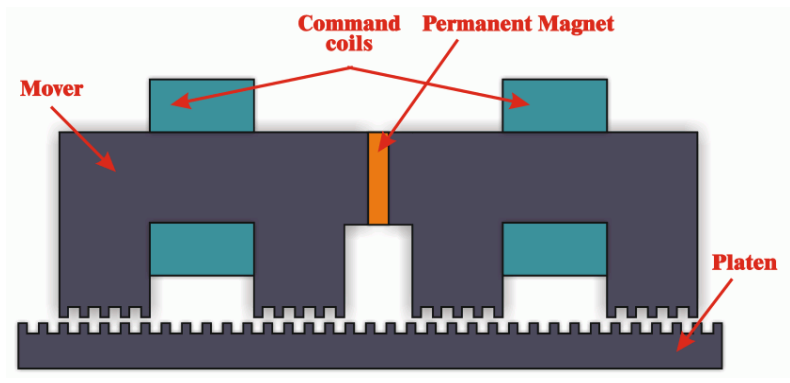

Fig. 1. The classical hybrid linear stepper motor.

This linear motor was at that time one of those having the highest force density. But it had several disadvantages. In any of the mover's position one of its poles was generating a significant breaking force, diminishing the total tangential force produced by the motor and thus reducing its efficiency. Beside this the magnetic flux passing through both the mover and the platen gave rise to a very strong normal force of attraction between the two armatures. This was over 10 times the peak holding tangential force of the motor. Due to this high attractive force produced of all the poles sophisticated linear bearing systems are required to maintain the precise air-gap between the mover and platen.

Two notable solutions were suggested in the past years to overcome the disadvantages of the classical hybrid linear motors. A new high precision permanent magnet linear motor structure (see Fig. 2) was proposed by the designers working at Shinko Electric Co. (Japan) [5].

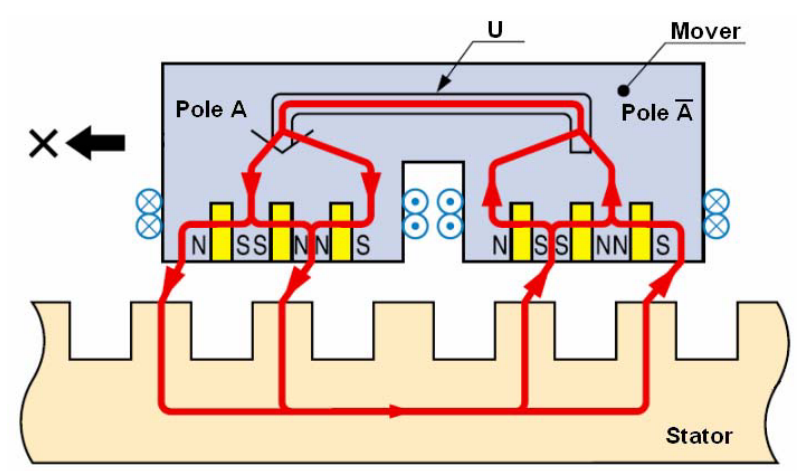

Fig. 2. The High Power Density (HD) Linear Motor. 
The new magnetic circuit structure with permanent magnets of the High Power Density (HD) Linear Motor (given in Fig. 2) made possible to increase its power density. Several small permanent magnets are set in the slots of the mover's poles. By its proper design it become possible that all the poles on the mover to generate tangential force at any moment of its movement.
Another improved variant of the hybrid linear stepper motor was designed by the researchers from Technical University of Cluj (Romania) [6].

The double salient permanent magnet linear motor given in Fig. 3a is built up of high force modules (as that shown in Fig. 3b).

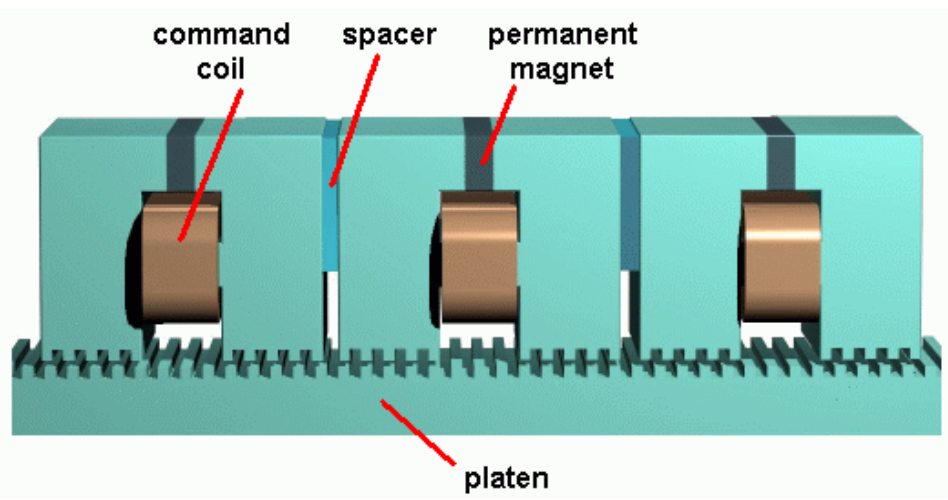

a)

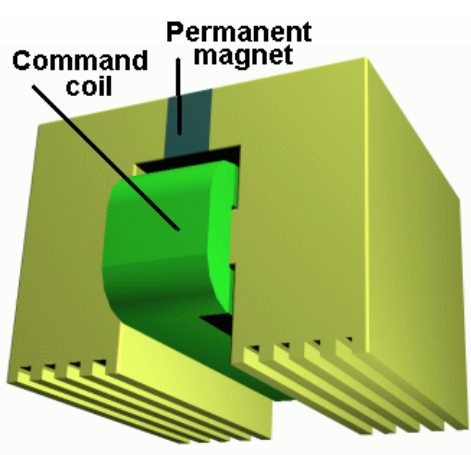

b)

Fig. 3. The modular double salient permanent magnet linear motor.

Each module has a very simple construction: two teethed salient poles separated by a permanent magnet generating the excitation field. On a core branch placed under the permanent magnet a command coil is wound.

If the command coil is not energized the magnetic flux generated by the permanent magnet passes almost entirely through the core branch on which the command coil is wound. In this case the module can be considered as inactive. Practically there is neither normal, nor tangential force produced by it.

Feeding the command coil a command flux is produced which will force the flux of the permanent magnet to pass through the air-gap and to generate significant forces.

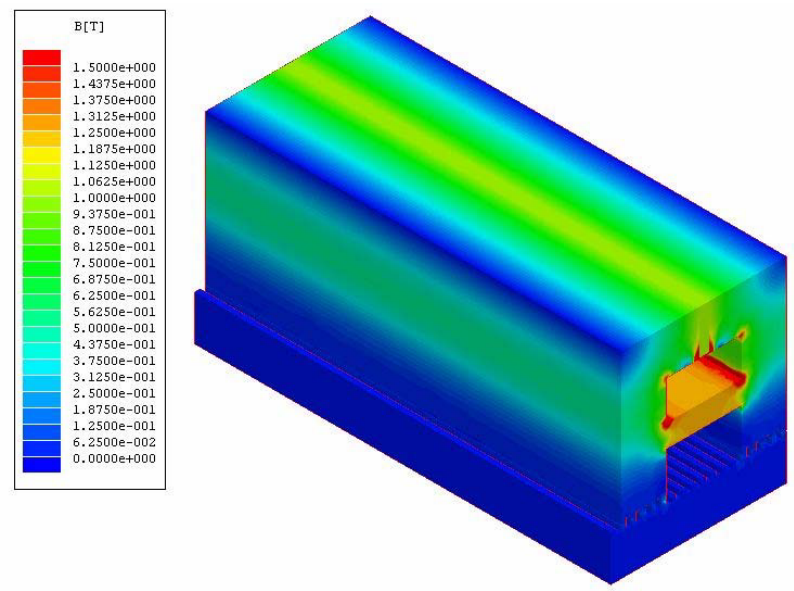

a) Passive mover
Hence at one moment only the active force will generate tangential and normal forces, the other modules remain inactive. Therefore the main disadvantage of the classical hybrid stepper motor was eliminated.

The novel structure, the design, the simulation and the control of this linear machine was presented in several international conferences. Thus here only some never published results obtained via the 3D FEM (finite elements method) based magnetic field analysis will be given. Only a single module and the stator portion under it were analyzed. In Fig. 4 the shaded plots of the flux density distribution through the motor are given for the two distinguish cases: when the mover is passive, and when its command coil is energized.
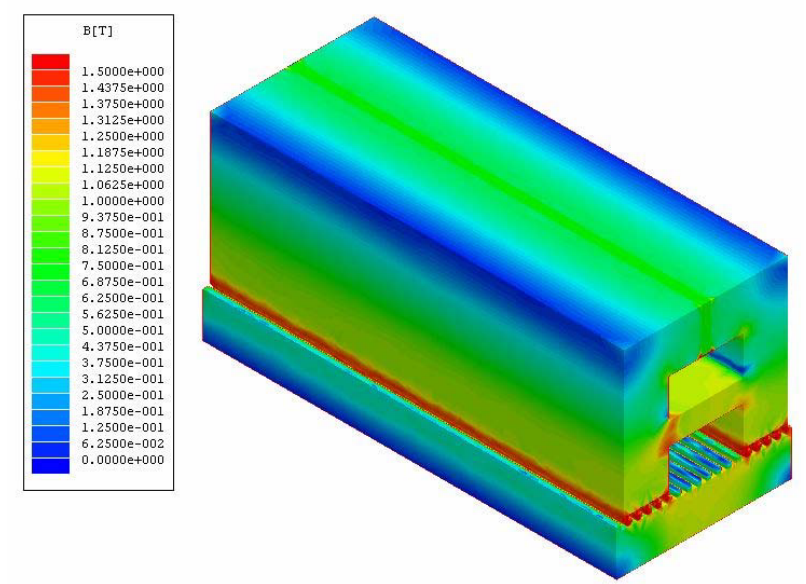

b) Active mover

Fig. 4. Results of the 3D FEM analysis of the double salient permanent magnet linear motor.

As it can be seen from these results the working principle of the double salient permanent magnet linear motor is proved also by means of the 3D FEM simulation. Hence when the command coils of the mover are not energized the greatest flux density can be observed in the core branch placed between the two poles, indicating that the main part of the magnetic flux generated by the permanent magnet is passing through this core element and not through the air-gap of the machine (see Fig. 4a). 
The flux density distribution given in Fig. $4 \mathrm{~b}$ shows that when the command coil is properly energized the foremost part of the magnetic flux is forced through the air-gap, exactly as it was theoretically expected.

\section{The Novel Modular Linear Machine Topology}

The electrical machines have various topologies. In general terms the iron core carries magnetic flux around the windings of the machine in order to create an electromotive force. The magnetic flux can pass in a direction parallel, or mainly perpendicular to the direction of motion (see Fig. 5). In the first case, the machine is said to be longitudinal, and in the second case transverse [7].

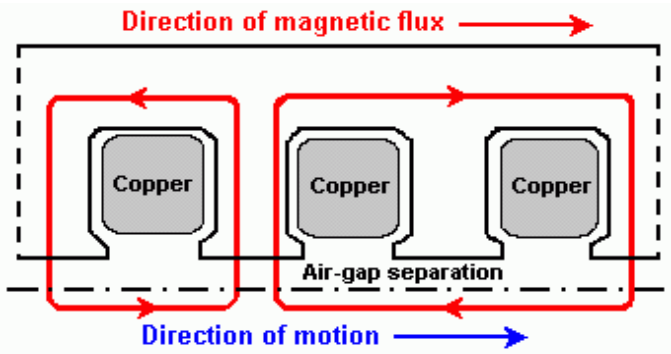

a) Longitudinal

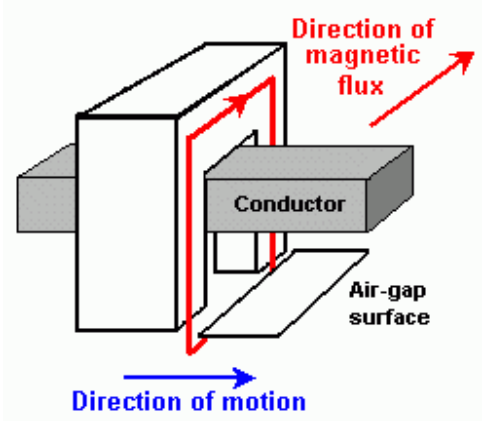

b) Transversal

Fig. 5. Magnetic flux orientation in electrical machines.

The linear machine structure to be presented also has the magnetic flux path transversal to the direction of its movement. It resulted by combining the modular structure of the double salient permanent magnet linear motor given in Fig. 3 with a linear variant of a transverse flux machine (TFM) with permanent magnets on the stator and passive rotor [8].

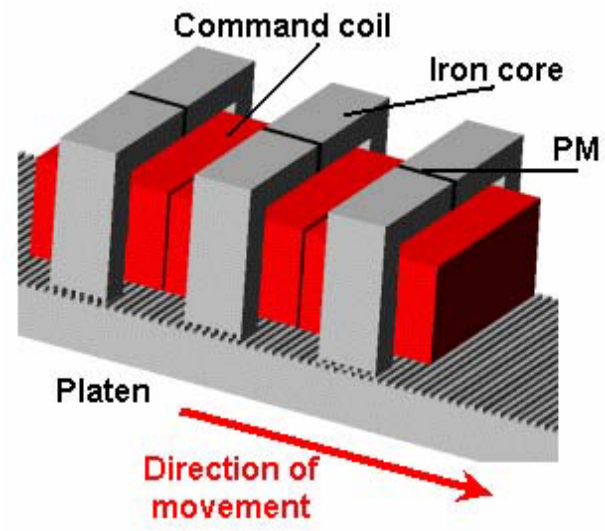

Fig. 6. The proposed modular linear machine.
The single-sided three-phase variant of the proposed linear motor structure is given in Fig. 6. A three-phase (three module) variant was selected because of the easy implementation of the control strategy on general purpose three-phase power converters.

The iron core of the module is shown in Fig. 7. It is very similar to the iron core of the module given in Fig. 3b. The single basic difference is that the poles of this variant are toothed in the transverse direction.

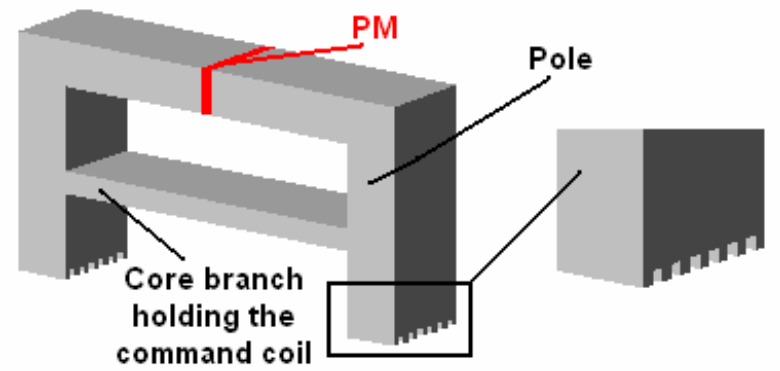

Fig. 7. The iron core of the mover.

The working principle of the machine is explained in Fig. 8 and it is identical with that of the linear motor given in Fig. 3. When the module is passive the flux generated by the permanent magnet closes mostly inside the mover's iron core.

When the command coil is energized, the magnetic flux produced by the winding practically enforces the flux of the permanent magnet through the air-gap, generating this way tangential and normal forces in a manner as in the case of the double salient permanent magnet linear motor shown in Fig. 3.
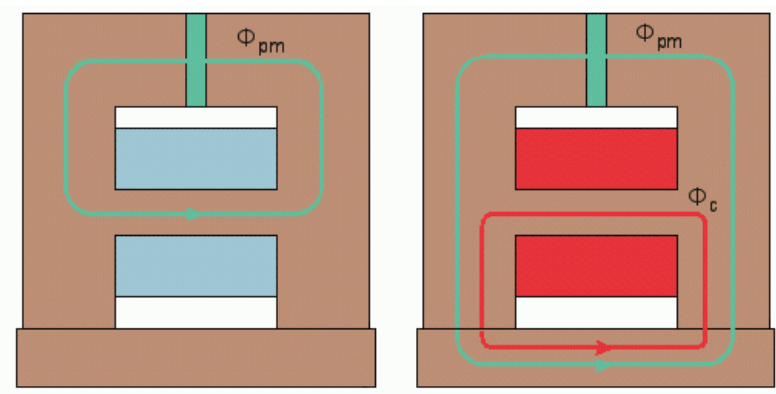

Fig. 8. The working principle of the linear machine.

The proposed linear machine structure is in fact a variable reluctance machine. Its movement is possible only if the modules are shifted by a third of the teeth pitch. Energizing the command coil of one module its teeth will be aligned with the teeth of the platen. By sequential feeding of the command coils continuous linear movement of any direction can be assured $[6,9]$.

The detailed design procedure of the proposed linear machine was presented in previous papers [9].

Here only the main dimensions of the sample motor's modules to be further analyzed will be given. Fig. 9 shows both the lateral and frontal view of the modules used in the single-sided variant of the proposed linear motor [9]. 


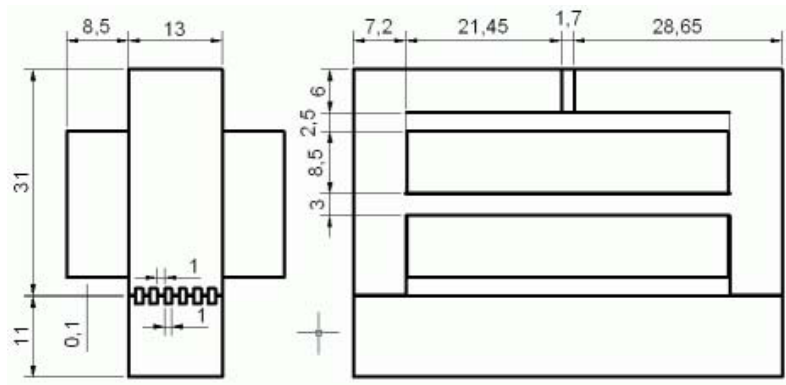

Fig. 9. Main sizes of the modules.

\section{3D FEM ANALYSIS OF THE LINEAR MOTOR}

The designed structure of the proposed modular linear machine was analyzed by means of three-dimensional (3D) finite elements method based numerical magnetic field computation.

As in any time moment only a single module is active in the linear machine it was enough to analyze only a single module and the portion of the stator under it (as shown in Fig. 10).

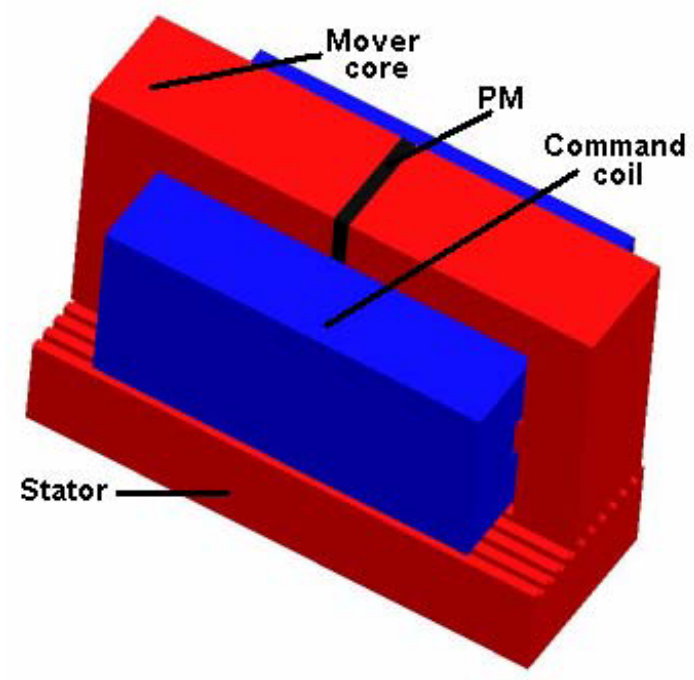

Fig. 10. The analyzed motor structure.

The frontal and lateral view of the analyzed motor part is given in Fig. 11 for better understanding of the problem to be solved.
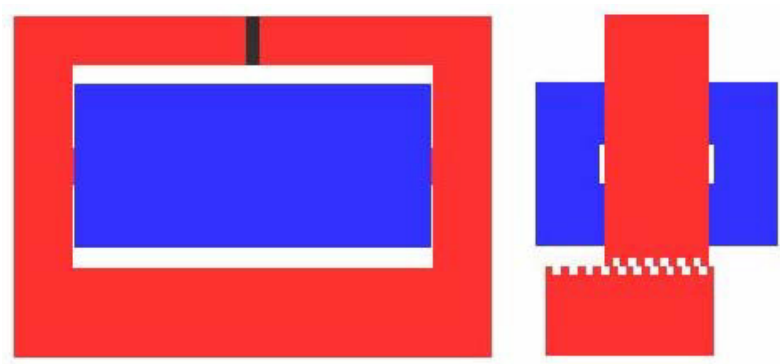

Fig. 11. Two views of the analyzed motor structure.

The three-dimensional mesh generated over the analyzed structure is given in Fig. 12.

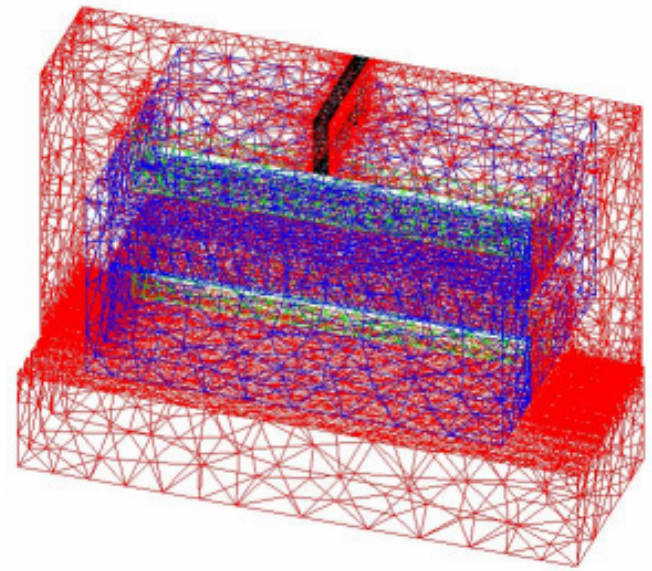

Fig. 12. The 3D mesh generated.

Next results of the 3D field computation performed for the presented motor structure are given. First see the field density distribution in the two cases in study: when the teeth on the stator and on the mover are aligned and the command coil is not fed (Fig. 13a), respectively when the mover is at a third of the teeth pitch respectively to the stator and its command coil is energized (Fig. 13b).

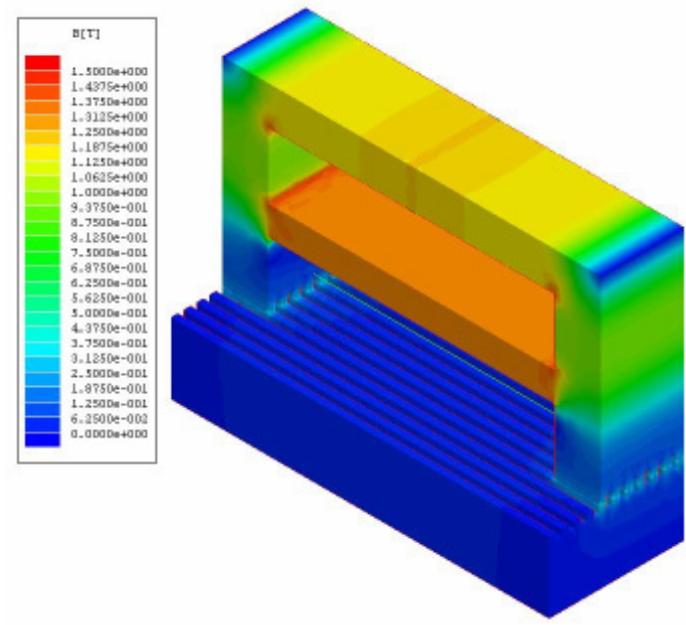

a) passive module

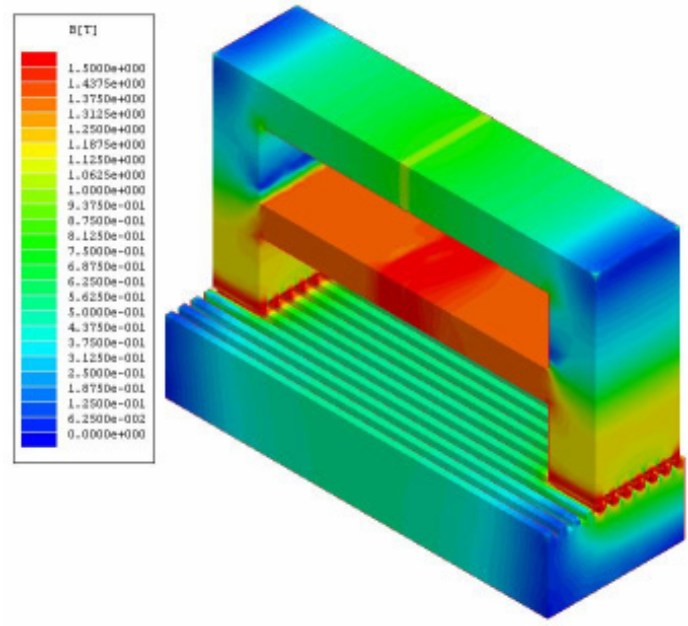

b) active module

Fig. 13. The flux density distribution obtained via 3D FEM analysis. 
In a similar manner to the cases given in Fig. 4 the working principle of the modular linear motor was proven also in this case, when the teeth of the mover are axially to the permanent magnet's magnetization direction.

The tangential and normal forces computed in the case when the module was active are the following: $F_{t}=15.12 \mathrm{~N}$ and $F_{n}=93.17 \mathrm{~N}$. As it can be observed the normal attractive forces between the two armatures are very great, over 6 times greater than the useful tangential tracking force developed by the module. In order to reduce the high normal forces a double-sided variant of the motor will be described and analyzed.

\section{The Double-Sided VARIANT OF THE Linear Motor}

To balance the high normal forces acting between the armatures a double-sided variant of the above-presented motor was proposed, designed and analyzed.

Practically the double-sided variant is composed of two motors such as that given in Fig. 6. The upper sides of two modules are coupled together. The two command coils are series-connected. In the middle part of the motor is placed the permanent magnet. The new mover assembly can be displaced between two parallel stators teethed on their internal part, as it can be seen in Fig. 14.

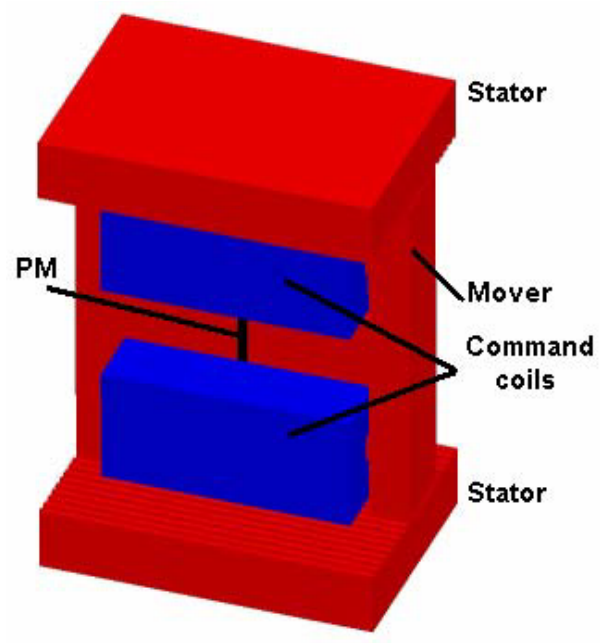

Fig. 14. The analyzed structure of the double-sided modular linear motor.

Also in this case two views of the combined motor structure are given in Fig. 15 for better understanding the placement of the different motor components.
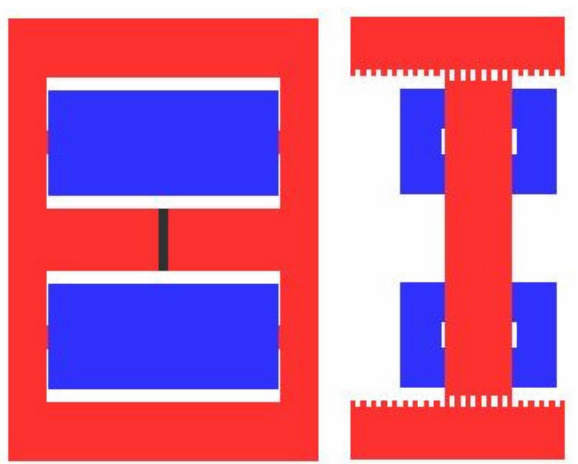

Fig. 15. Two views of the analyzed double-sided modular linear motor structure.
The 3D field computations were performed also for the double-sided version of the modular linear motor. Just as in the above presented cases two situations were studied: when the teeth on the stator and on the mover are aligned and the command coil is not fed (passive module), respectively when the mover is displaced by a third of the teeth pitch from this position and the command coil is energized (active module). The obtained flux density distributions in these two cases are given in Fig. 16.

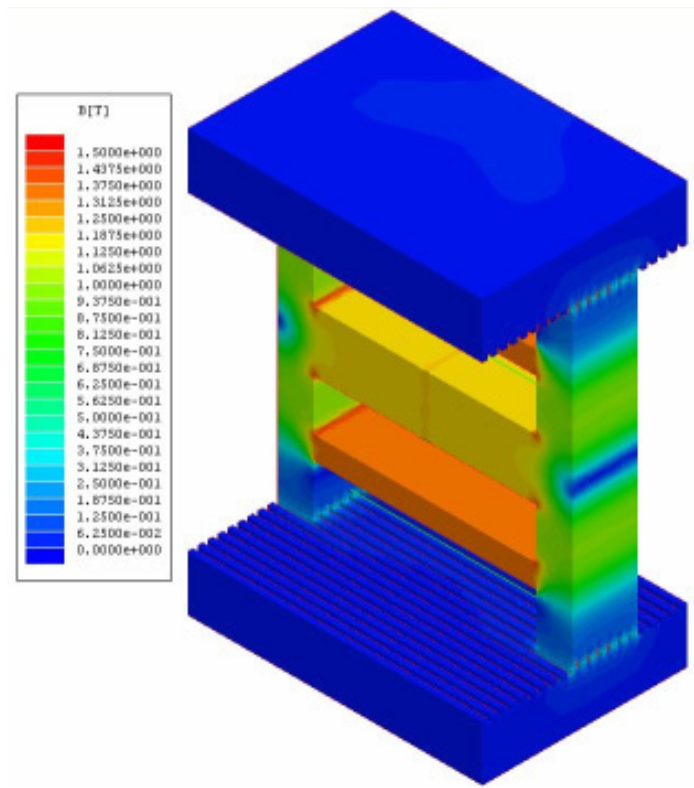

a) passive module

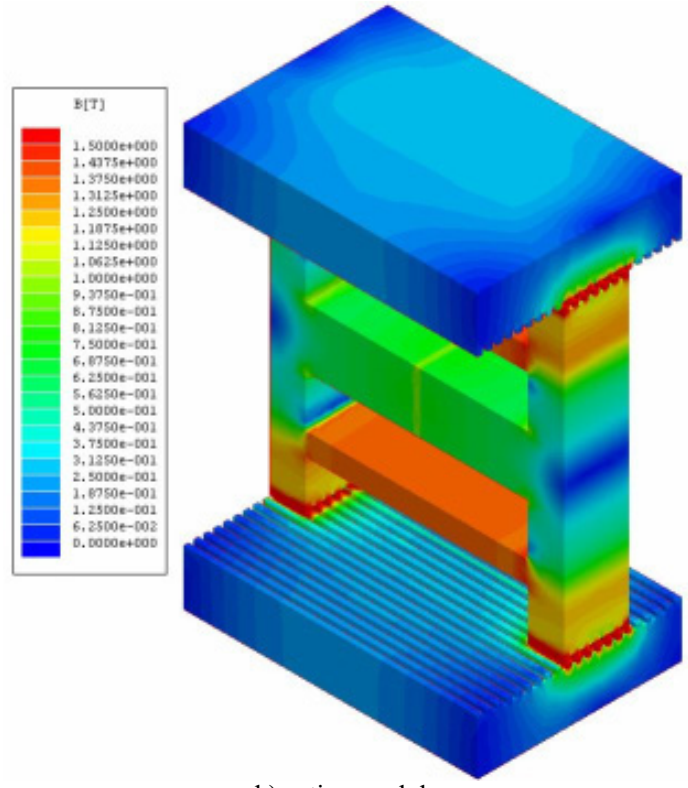

b) active module

Fig. 16. The flux density distribution in the double-sided variant of the modular linear motor.

As it can be seen from the results given in Fig. 16 the working principle of the modular linear motor described above is applied also in this case: almost no magnetic flux is passing through the air-gap when the command coil is not fed, and by energizing the command coil the flux generated by the permanent magnet is forced through the air-gap generating both tangential and normal forces. 
The forces computed in this case for the active module are in accordance with the expectations: $F_{t}=30.42 \mathrm{~N}$ and $F_{n}=6.21 \mathrm{~N}$. It means that the tangential force was doubled due to the combined structure of the machine and the normal force is very low because it is balanced by the existence of the two parallel stators.

This double-sided modular linear motor can be applied in several industrial applications where precise positions are required. Next an interesting linear positioning system using two such modular linear machines will be presented.

\section{LineAR Positioning SySTEM USING DOUBLE-SidED MODULAR LINEAR MOTORS}

The key of automated or semi-automated production lines are the conveyors carrying the parts from one processing tool to another [10]. Hence the individual parts can be moved through the automated factory for routine processing, leaving workers free for quality control tasks or for other higher responsibilities. Conveyors also proved to be useful in transporting heavy or hazardous products, reducing worker injuries.

Several conveyor systems are used in industry: skatewheels, belts, live rollers, etc. All of these are driven by rotational electrical machines coupled to inefficient rotary-to-linear movement conversion devices.

Much more efficient solution is to use linear motor driven conveyors, due to the lack of any mechanical conversion device. These conveyors are easy to control by simple variable-speed drivers and have practically a lifetime durability.

The proposed linear direct driven conveyor system is composed by two double-sided modular linear motors placed in parallel. On each motor two together fixed movers can be displaced. The two mover assemblies of a single motor are mechanically connected together by the carrier on which the parts to be transported can be fixed (see Fig. 17).

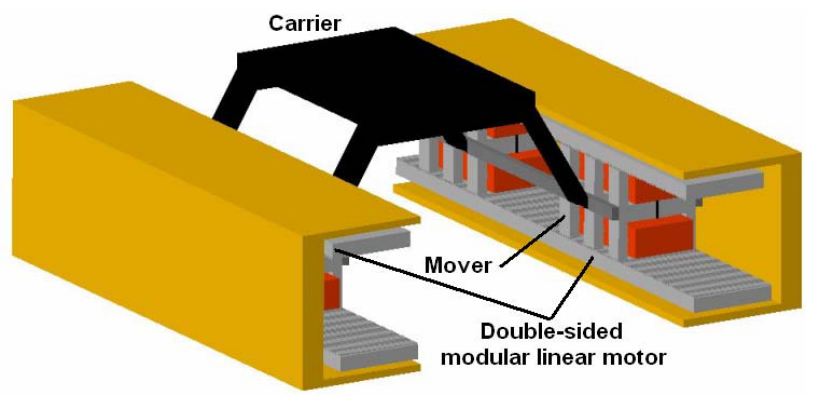

Fig. 17. The proposed industrial linear positioning system using double-sided modular linear motors.

The conveyor system shown in Fig. 17 can be built up of any length. As the stator of the linear machines is passive in the same system also more than one carrier can move at the same time. Of course in this case an adequate control system must be applied to avoid collision of the carriers [11].

The proposed industrial linear positioning system due to its direct driven structure can achieve fast speeds and high accuracy. Hence it can be used also in high-tech industries (for example in semiconductor wafer handling and processing equipment, etc.).

\section{CONCLUSIONS}

The iron-core permanent magnet linear motors are of great interest in many fields of industrial automation due to their high thrust to weight ratio and good dynamic performances even with heavy loads.

Such machines are the modular linear machines presented in this paper. They can be easy manufactured and have relatively low production costs, since the price of the permanent magnets has a decreasing tendency. Its modular structure enables to easy adjust the motor performances to the user's requirements without substantial changes in its basic structure. They are simple to control by unipolar current pulses.

The proposed double-sided construction can eliminate the main drawback of the linear machines: the high attractive forces between the iron cored armatures. The above presented precise linear positioning system using such linear motors seems to be a good solution for advanced flexible manufacturing systems.

\section{ACKNOWLEDGEMENTS}

The work was possible due to the support given by the Romanian National Council of Scientific Research in Higher Education under grants A363/2003, A369/2003, A769/2005 and TD257/2005. The authors should like to sincerely thank this way for the financial support.

\section{REFERENCES}

[1] Boldea, I., "Linear Electromagnetic Actuators and their Control: A Review," Proceedings of the $10^{\text {th }}$ International Power Electronics and Motion Control Conference (EPE-PEMC '2002), Cavtat \& Dubrovnik (Croatia), 2002, on CD: KEY-01.pdf.

[2] Szabó, L., Viorel I.A., Iancu V., Popa, D.C., "Soft Magnetic Composites Used in Transverse Flux Machines," Oradea University Annals, Electrotechnical Fascicle, 2004, pp. 134-141.

[3] Cassat, A., Corsi, N., Moser, R., Wavre, N., "Direct Linear Drives: Market and Performance Status," Proceedings of the $4^{\text {th }}$ International Symposium on Linear Drives for Industry Applications LDIA'2003, Birmingham (UK), 2003, on CD: SP1.pdf.

[4] Viorel I.A., Szabó L., "Hybrid Linear Stepper Motors," Mediamira, Cluj (Romania), 1998.

[5] "HDE-A Motor Catalogue," Shinko Electric Co. Ltd., Tokyo

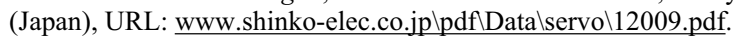

[6] Szabó, L., Viorel, I.A., Chişu, I., Kovács, Z., "A Novel Double Salient Permanent Magnet Linear Motor," Proceedings of the International Conference on Power Electronics, Drives and Motion (PCIM), Nürnberg (Germany), vol. Intelligent Motion, 1999 , pp. 285-290.

[7] Dubois, M.R., "Optimized Permanent Magnet Generator Topologies for Direct-Drive Wind Turbines," Ph.D. Thesis, TU of Delft, Holland, 2004.

[8] Viorel, I.A, Henneberger, G., Blissenbach, R., Löwenstein, L., "Transverse Flux Machines. Their Behaviour, Design, Control and Applications," Mediamira, Cluj (Romania), 2003.

[9] Popa, D.C., Iancu, V., Viorel, I.A., Szabó, L., "C.A.D. of Linear Transverse Flux Motors," Annals of the Polytechnic Institute of Iaşi, vol. LI (LV), fasc. 5, Electrotechnics, Energetics and Electronics, 2005, pp. 79-84.

[10] Szabó, L., Viorel, I.A., Szépi, I., "Linear and Planar Variable Reluctance Motors for Flexible Manufacturing Cells," Advances in Electrical and Electronic Engineering (Slovakia), no. 2, vol. 3 , 2004, pp. 39-42.

[11] Szabó, L., Viorel, I.A., Dobai, B.J., Szépi, I., "Optimal Trajectory Generation for a Modular Planar Motor Used in Flexible Manufacturing Systems," Proceedings of the $11^{\text {th }}$ International Power Electronics and Motion Control Conference (EPE-PEMC '2004), Riga (Lithuania), 2004, on CD: A53272.pdf. 